ment of capacity for preparedness and response; (4) provision of technical guide for structural integrity of health facilities; and (5) advocacy and awareness of safe health facilities campaign. Target countries included Cambodia, Lao PDR, Philippines, and Vietnam.

Conclusions: The strategy has provided positive results in terms of advocacy, technical resources on preparedness of hospitals, assessment of health facilities, and capability enhancement of health emergency managers at the national and local levels. Building strong infrastructures with a prepared staff that could remain functional during natural hazards is an integral part of disaster risk reduction. Advocacy, sharing of experiences, partnership with donors and private sectors, participation of civil society, and development of national policies will be enhanced as a next step. Keywords: Asia; disaster; health; preparedness; public health; risk reduction; Western Pacific

Prebosp Disaster Med

\section{Education and Training in Disaster Medicine in Belgium} J.B. Gillet; ${ }^{1}$ A. Meulemans, ${ }^{2}$ C. Lebaupin, ${ }^{2}$ J. Stroobants ${ }^{3}$

1. KU Leuven, Emergency Department, Leuven, Belgium

2. Clinique Saint Pierre, Ottignies, Belgium

3. AZ Middelheim, Antwerpen, Belgium

Introduction: In Belgium, as in many other countries, the courses of disaster medicine vary considerably in terms of objectives, basic requirements, acquired skills, learning methods, and content. The lack of coherence leads to two types of problems:

1. The various trainings do not supply skills and qualifications at the same level; and

2. The authorities cannot use the acquired skills and knowledge as functioning standards.

Methods: Representatives of the seven Belgian universities and representatives of scientific and professional societies in emergency medicine came together to:

1. Make an inventory of the existing training programs; and

2. Define a common vision for the future.

Results: A consensus was agreed upon to determine four levels of education:

1. A basic level for each relief professional in different disciplines. The objective is to allow those professionals to understand the organization of emergency medical services and to collaborate with it: theoretical course of four hours;

2. A basic level for doctors and specialized nurses working in an emergency department. The objective is to train them in case they are faced with a large number of victims, pending the arrival of reinforcements: 20 hours, with a field exercise;

3. A basic level for emergency physicians and nurses for the responsibility function of operational director of emergency medical services including risk assessment: 15 days of courses with a tabletop exercise, a field exercise, and an evaluation; and

4. A higher level for doctors in education, research, and risk analysis: Master after Master: one year.

Conclusions: It was decided unanimously that harmonization of training is necessary in Belgium. A large medical consensus was researched to keep four levels of education and to harmonize the contents and duration of the different trainings.

Keywords: Belgium; disaster medicine; education; training

Prebosp Disaster Med

Bio-Terror Interactive Software and its Impact on the Knowledge of First Responders

Itay Fogel; Liat Tusk-Helerman; Ram Sagi

CBRN Medicine Branch, Medical Corps Headquarters, Israel

Defense Forces, Israel

Preparedness for a bioterrorist attack depends, among other things, on the early identification of the event by a group of sentinels, which by definition, includes physicians, paramedics, and nurses who first encounter affected individuals.

Data indicated that the knowledge and awareness level of medical staff regarding bioterrorist agents is low- a fact that may have a negative impact on the speed with which the agents are identified, and thus, the ability to affect the course of the diseases they cause.

In order to improve the knowledge of medical staff regarding bioterrorism agents, interactive software was developed and into four units, each one dealing with one of the four bioterrorism agents: (1) anthrax; (2) plague; (3) smallpox; and (4) botulinum.

The software was designed for use by medical personnel in hospitals or community clinics, and features a convenient and user-friendly work interface that independently manages clinical cases and provides feedback. Similarly, the software was designed for use in any large institution: )hospitals, military medical schools, etc.), so it can be used at a time that is convenient for the attending physician, in almost any place without the need for a trained instructor.

The results of surveys and tests administered among physicians and paramedics who sampled the software at the military medical school indicate substantial student satisfaction with the new learning method as well as a significant increase in knowledge regarding bioterrorism agents.

Since this learning tool is intended to increase knowledge and awareness among physicians and paramedics, we anticipate that the guide ultimately will improve the preparedness of the medical system for a bioterrorist attack.

Keywords: bioterrorism; computer; first responders; software Prehosp Disaster Med

\section{Managing Surge Capacity-Lessons Learned from a National, Mass-Casualty Simulation Exercise G.P.H. Choa; M.K.F. Leong \\ Singapore General Hospital, Singapore}

Introduction: Surge capacity is a challenge that all emergency departments face during a mass-casualty situation. A full-scale, mass-casualty simulation exercise at Singapore General Hospital and the lessons learned will be presented. The hospital's experience in creating temporary intensive care unit beds and the presence of a forward deployed anesthesist and surgeon at the emergency department also will be presented.

Methods: A real-time simulation exercise with trained volunteers acting as casualties was conducted by the Ministry of Health. The hospital had to submit its mass-casualty 